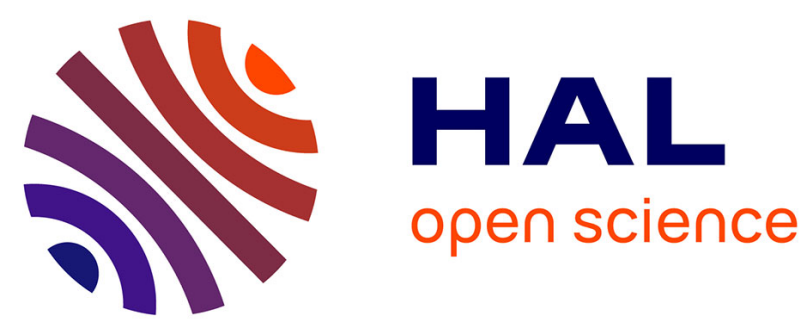

\title{
Chapitre 9. Évaluation réflexive en formation professionnelle et évaluation interactive dans les classes
}

Françoise Campanale

\section{To cite this version:}

Françoise Campanale. Chapitre 9. Évaluation réflexive en formation professionnelle et évaluation interactive dans les classes. L. Allal; L. Mottier-Lopez. Régulation des apprentissages en situation scolaire et en formation., De Boeck Université, pp. 191-206, 2007, 10.3917/dbu.motti.2007.01.0191 . hal-01172743v2

\section{HAL Id: hal-01172743 \\ https://hal.science/hal-01172743v2}

Submitted on 9 Jul 2015

HAL is a multi-disciplinary open access archive for the deposit and dissemination of scientific research documents, whether they are published or not. The documents may come from teaching and research institutions in France or abroad, or from public or private research centers.
L'archive ouverte pluridisciplinaire HAL, est destinée au dépôt et à la diffusion de documents scientifiques de niveau recherche, publiés ou non, émanant des établissements d'enseignement et de recherche français ou étrangers, des laboratoires publics ou privés. 


\section{снитгвв 9}

EVALUATION RÉFLEXIVE

EN FORMATION PROFESSIONNELLE

ET ÉVALUATION INTERACTIVE

DANS LES CLASSES

Françoise Campanale

Les savoirs en ce qui concerne l'évaluation formative, privilégiant une approche réflexive, sont maintenant largement diffusés par de nombreux articles et ouvrages, ainsi qu'à travers des formations. Des modalités d'évaluation mutuelle entre élèves, d'autoévaluation à l'aide de grilles de critères, de questionnaires-guides pour l'effectuation de tâches complexes, ont montré leur efficacité en matière de régulation des apprentissages (Allal, Bain \& Perrenoud, 1993 ; Romainville, 1993). Or, ces modalités sont encore peu utilisées dans les classes. L'évaluation formative reste une « utopie porteuse » (Hadji, 1997).

En faire une réalité dans le quotidien de la classe supposerait, pour la plupart des enseignants en exercice, de modifier leurs pratiques évaluatives. Les pratiques ${ }^{1}$ s'inscrivent dans les coutumes et croyances des groupes sociaux (Prost, 1985). Elles sont constituées de savoir-faire, acquis la plupart du temps de façon implicite, sans connaître les raisons (Fayol, 1998), de routines difficilement verbalisables, et de

\footnotetext{
${ }^{1}$ On désigne généralement par pratiques ce qui relève du faire. Pour De Montmollin (1996), elles sont constituées de savoir-faire, de routines difficilement verbalisables, et de métaconnaissances qui sont caractéristiques de l'expérience.
} 
Campanale, F. (2007). Evaluation réflexive en formation professionnelle et évaluation interactive dans le 8 classes. In L. Allal \& L. Mottier-Lopez (dir.), Régulation des apprentissages en situation scolaire et en formation (pp. 191-206). Bruxelles : De Boëck.

métaconnaissances qui sont caractéristiques de l'expérience (De Montmollin, 1996). Les modifier passe à la fois par une réorientation du système de représentations qui les fonde et une prise de conscience de sa façon de pratiquer pour la réguler en fonction d'une nouvelle orientation. Cela ne peut se faire qu'à travers un processus de développement personnel dans un contexte de socialisation, de formation (Vanderberghe, 1986).

Ce chapitre traite de la formation continue des enseignants ${ }^{2}$ à l'évaluation des apprentissages. Il fournit des éléments de réponse à la question : A quelles conditions une action de formation continue contribue-t-elle à ce que les enseignants mettent en œuvre dans les classes de l'évaluation interactive pour réguler apprentissages et enseignement?

Seront successivement abordés :

- les facteurs favorisant des changements de pratiques ;

- les processus autoévaluatifs susceptibles de générer des décisions de modification, de régulation de pratiques ;

- les situations qui peuvent provoquer ces processus ;

- les problèmes liés aux contextes de travail.

L'hypothèse, qui sous-tend ce chapitre, est que les pratiques développées en formation sont aussi déterminantes que le contenu pour réguler les pratiques mises en œuvre en classe. L'évaluation interactive serait à la fois objet et vecteur de formation.

\section{FACTEURS DE CHANGEMENTS DES PRATIQUES PÉDAGOGIQUES}

Des facteurs de changements de pratiques ont été mis en évidence par différents chercheurs, à partir d'entretiens avec des enseignants, d'études de cas de formation. J'en retiendrai trois qui interviennent aux différents niveaux de l'organisation générale de la formation, des modalités, de la posture du formateur.

\subsection{Une formation longue, distribuée, négociée}

C'est plutôt l'organisation distribuée sur un temps long, pour une équipe d'enseignants d'un même établissement, qui favorise le changement (Vandenberghe, 1986). Ce type d'organisation permet de préparer en formation des modalités pédagogiques à essayer en classe, de procéder lors de séances ultérieures de formation à des retours structurés sur ces essais, à des révisions, des abandons, à la préparation de nouveaux essais. Nous avons montré qu'une action de formation continue ne pouvait être véritablement efficace que sur au moins deux ans à raison

\footnotetext{
${ }^{2}$ A l'expression formation continue, utilisée en France, on préfère, dans d'autres pays, celle de développement professionnel.
} 
Campanale, F. (2007). Evaluation réflexive en formation professionnelle et évaluation interactive dans le3 classes. In L. Allal \& L. Mottier-Lopez (dir.), Régulation des apprentissages en situation scolaire et en formation (pp. 191-206). Bruxelles : De Boëck.

d'environ huit à neuf journées de formation (Campanale, 1996).

Le changement durable suppose une modification de l'habitus, «cette grammaire génératrice des pratiques » (selon l'expression de Bourdieu, reprise par Perrenoud, 1996b, p. 182), que le sujet ne connaît pas lui-même entièrement. A-t-on le droit de vouloir agir sur l'habitus d'un sujet ? La formation ne peut être qu'un processus volontairement engagé par le sujet. On ne peut que proposer et non pas imposer. Aussi, la formation continue s'inscrit-elle dans la négociation continue de ses objectifs et modalités.

\subsection{Une formation axée sur l'analyse des pratiques}

Qu'est-ce qui peut agir sur l'habitus ? A cette question, qu'il pose, Perrenoud (1996b) répond : de façon complémentaire, la transformation des conditions de la pratique et la prise de conscience de ses fonctionnements pour provoquer le passage de certaines actions sous le contrôle d'une référence explicite. Les conditions de la pratique, le formateur ne les maîtrise pas. Il peut toutefois réfléchir avec les enseignants sur les aménagements qu'ils sont libres d'effectuer, sur ceux qu'ils peuvent négocier avec l'administration de l'établissement (composition des équipes pédagogiques, aménagements d'emplois du temps, nombre de notes à fournir, modalités d'information des élèves et des parents sur les résultats scolaires...). La prise de conscience de ses fonctionnements appelle l'analyse des pratiques, une analyse qui articule pratique-théorie-pratique (Altet, 2000), dans une approche compréhensive éclairée par des savoirs pédagogiques et didactiques. L'analyse des pratiques est devenue une activité dominante dans la formation des enseignants. Il n'est d'ailleurs pas surprenant qu'une formation visant un changement de pratiques les prenne comme principal objet de travail.

Toutefois, les conceptions théoriques de l'analyse des pratiques et les modalités utilisées sont diverses. Dans une formation à l'évaluation, plus que sur des descriptions orales de pratiques, l'analyse peut s'appuyer sur des objets qui en sont des traces (formalisations de séquences, copies d'élèves, grilles d'évaluation...) que le formateur peut fournir, que les enseignants en formation veulent bien apporter et soumettre au travail collectif. Analyser des dispositifs d'évaluation des apprentissages en fonction des modèles de l'évaluation (Cardinet, 1986 ; Bonniol \& Vial, 1997) permet à la fois de s'approprier ces modèles théoriques et développe la capacité à analyser ses propres dispositifs. Au cours des séances de formation, l'analyse par les enseignants des objets qui témoignent de leurs pratiques évaluatives vise la prise de conscience par chacun d'eux du type d'évaluation qu'il met en oeuvre en classe, du rôle qu'il y joue, de celui qu'il donne aux élèves. Chacun peut alors identifier ce qu'il lui est possible de réguler dans sa propre pratique. Ainsi, l'analyse des pratiques évaluatives peut engager chacun dans un processus d'autoévaluation explicite, autrement dit d'évaluation réflexive. Mais, cet enjeu est fortement dépendant du crédit que les enseignants attribuent au formateur. 
Campanale, F. (2007). Evaluation réflexive en formation professionnelle et évaluation interactive dans les classes. In L. Allal \& L. Mottier-Lopez (dir.), Régulation des apprentissages en situation scolaire et en formation (pp. 191-206). Bruxelles : De Boëck.

\subsection{Un formateur crédible}

Pour Huberman (1986), la crédibilité du formateur tient à une double posture de pair et d'expert, à son appartenance à la même communauté professionnelle, mais avec un pied dans chaque univers, celui de la pratique et celui de la théorie. La posture du formateur d'adultes en formation continue n'est pas celle d'un enseignant avec des élèves. Certes, tout comme lui, il procède à une transposition didactique (Chevallard, 1991) des savoirs qu'il veut transmettre et organise leur appropriation à travers des activités ${ }^{3}$. Autrement dit, il provoque la transformation des savoirs issus de la recherche en "savoirs en usage ». Mais, il permet aussi la formalisation des savoirs tirés de la pratique (Malglaive, 1994). Il fait expliciter et analyser des dimensions non réfléchies de l'action, des routines (Perrenoud, 1999).

Dans une formation des enseignants à l'évaluation, le formateur évite de proposer «des prescriptions a prioristes qui ne tiennent pas compte de leurs expériences pratiques [celles des enseignants] et qui ne respectent pas le principe que l'enseignant, comme l'élève, doit construire ses stratégies d'action » (Allal, 1979/1985, p. 144). Il cherche avec les formés des réponses aux problèmes qu'ils rencontrent dans les classes, tout en fournissant des modèles interprétatifs. $\mathrm{Au}$ formateur, comme pair, les enseignants montrent les copies de leurs élèves, leurs préparations de séquences, et lui font part de leurs questions, voire de leurs déceptions. Il revient au formateur-expert de les aider à clarifier objectifs et critères d'évaluation, à questionner la cohérence entre tâches demandées aux élèves et objectifs d'apprentissage, de proposer de reconstruire ensemble les séquences pour y introduire des modalités qui impliquent les élèves dans l'évaluation. Le travail se fait alors collaboratif. Chacun y contribue pour ce qui relève de ses compétences : le formateur en ce qui concerne l'évaluation, les enseignants en ce qui concerne la didactique de leur discipline et leur connaissance du contexte dans lequel sera mise en œuvre la séquence. Cette collaboration permet aux enseignants de faire valoir leurs compétences et de relativiser l'expertise du formateur ${ }^{4}$. Il est tentant de rapprocher ce type de relation de ce qui ressort des travaux sur l'influence sociale dans le travail collaboratif. Une expertise ressentie comme trop dominante peut menacer l'identité professionnelle des formés et provoquer des résistances défensives, alors qu'une expertise non menaçante favoriserait au contraire l'apprentissage et le transfert (Butera, Gardair, Maggi \& Mugny, 1998 ; Mugny, Quiamzade, Pigière, Dragulescu \& Buchs, 2002).

\footnotetext{
${ }^{3}$ Voir par exemple le matériel pour la formation continue à l'évaluation formative proposé par Allal (1991).

${ }^{4}$ Perrenoud (1996b) évoque l'importance de la manifestation des hésitations et du doute par le formateur pour faciliter l'acceptation du risque de changement par les enseignants.
} 
Campanale, F. (2007). Evaluation réflexive en formation professionnelle et évaluation interactive dans le classes. In L. Allal \& L. Mottier-Lopez (dir.), Régulation des apprentissages en situation scolaire et en formation (pp. 191-206). Bruxelles : De Boëck.

Ces conditions constituent un cadre propice au développement professionnel visant des régulations de pratiques pédagogiques. Toutefois, des routines évaluatives, ancrées par des années d'expérience professionnelle, ne se transforment pas facilement, d'autant plus que ce changement met en danger tout l'équilibre du système pédagogique (Perrenoud, 1993). La formation devrait permettre d'acquérir des références théoriques et pratiques, nécessaires à une autoévaluation explicite de sa propre pratique, à l'identification de ce qu'on peut changer, à la détermination de moyens pour les transformations envisagées.

\section{PROCESSUS AUTOÉVALUATIFS ET RÉGULATIONS}

C'est à l'autoévaluation explicite que nous nous intéressons, celle qui peut générer des régulations conscientes. Qu'entendons-nous par autoévaluation explicite? Quels processus sont en jeu? Nous reprenons ici un modèle de l'autoévaluation (Campanale, 1997, 1998, 1999), qui peut éclairer situations et phénomènes observés en formation ${ }^{5}$.

\subsection{L'autoévaluation explicite : une confrontation tripolaire}

L'activité intentionnelle combine trois phases interactives (anticipation, réalisation, contrôle), pilotées par un réseau ramifié de représentations, représentations de l'action et de son but, du milieu dans lequel s'effectue l'action, des moyens et des opérations nécessaires à sa réalisation (Talyzina, 1980). En retour, ce réseau de représentations, que nous nommerons référentiel d'action interne, se modifie sous l'effet du feedback renvoyé par ce que produit l'activité. Référentiel interne et activité se régulent l'un par rapport à l'autre. Piaget distingue la régulation active de l'action des régulations automatisées, liées au tâtonnement, parce que la régulation active requiert des choix «qui supposent [...] une estimation pour ou contre $\gg(1974 a$, p. 115). Autrement dit, la régulation active suppose une mise sous contrôle par le sujet de son activité et de ce qui la génère. Elle découle d'une autoévaluation consciente, explicite (au sens d'explicitable au moins pour soi), de son action (produit, démarche utilisés/envisagés).

Ce surgissement de l'autoévaluation explicite, qui se traduit par un langage intérieur $^{6}$, un autoquestionnement (Vial, 1995), passe par une prise de distance du sujet d'avec son activité. Qu'est-ce qui peut provoquer cette prise de distance ? Un résultat complètement inattendu, qui nous fait nous questionner; le temps qui passe et qui fait qu'on regarde différemment ce qu'on a fait antérieurement ; l'intervention d'un tiers, dont les interprétations nous révèlent autrement notre action. Ce qui modifie le référentiel qui a guidé l'action du sujet, ce sont des «interactions

\footnotetext{
${ }^{5}$ Ce modèle a permis d'analyser des situations d'autoévaluation en formation continue d'enseignants. Il peut être utilisé aussi pour les situations d'autoévaluation organisées pour des élèves.

${ }^{6}$ Ce contrôle cognitif de l'action est un des rôles du langage intérieur (Vygotsky, 1985).
} 
Campanale, F. (2007). Evaluation réflexive en formation professionnelle et évaluation interactive dans le( classes. In L. Allal \& L. Mottier-Lopez (dir.), Régulation des apprentissages en situation scolaire et en formation (pp. 191-206). Bruxelles : De Boëck.

(immédiates ou différées, effectives ou symboliques) [...] avec son environnement matériel et social » (Allal, 1999b, p. 43). Nous savons comme les remarques d'autrui nous sont précieuses pour déceler les imprécisions du texte qu'on vient d'écrire et qui ne nous apparaissent pas à la seule relecture. Nous savons comme la lecture plurielle de situations, les interprétations croisées, enrichissent l'évaluation tout en interrogeant les référentiels de chacun des évaluateurs (Ardoino, 1993), en les engageant dans une réflexion métacognitive (Noël, Romainville \& Wolfs, 1995). Ainsi, autoévaluer explicitement suppose la prise en compte de références externes (consignes, grille de critères, modèle théorique, interprétations fournies par des pairs et/ou le formateur), qui viennent altérer $^{7}$ (Campanale, 1996, 1997) le référentiel interne du sujet. Le sujet peut alors regarder autrement les objets qui témoignent de son activité (productions/démarche explicitée, expression de représentations) et du même coup décider de la réguler.

Comme le montre la figure 1, l'autoévaluation s'accomplit dans cette confrontation tripolaire (référentiel interne - référents externes - objets évalués) et suscite des régulations actives qui touchent le référentiel d'action interne et l'activité concrète.

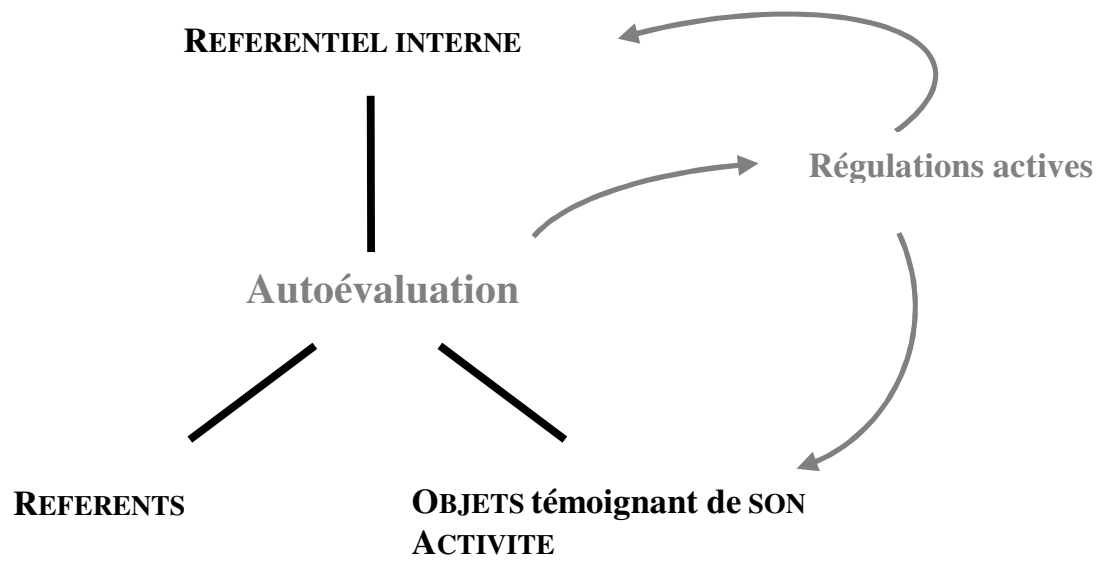

Figure 1 - Processus d'autoévaluation explicite

\subsection{Différents processus de prise de distance}

Lors d'un travail de recherche antérieur (Campanale, 1996), j'ai constaté que des enseignants en formation, mis en situation d'autoévaluation de leur pratique, manifestaient des prises de distance d'ampleur et de focalisation différentes. La réflexion sur l'action est en général régressive, de la périphérie au centre. Autrement

\footnotetext{
${ }^{7}$ Altération est à prendre ici au sens propre de devenir autre.
} 
Campanale, F. (2007). Evaluation réflexive en formation professionnelle et évaluation interactive dans le\$ classes. In L. Allal \& L. Mottier-Lopez (dir.), Régulation des apprentissages en situation scolaire et en formation (pp. 191-206). Bruxelles : De Boëck.

dit, de la comparaison des résultats avec le but, le sujet remonte à l'analyse des moyens employés pour réaliser l'action, et enfin au pourquoi des choix (Piaget, 1974 a et b). J'ai alors distingué trois processus de prise de distance, qui peuvent ou non se combiner : le recul, la distanciation, la décentration.

Comment savoir s'il y a eu autoévaluation? On peut interroger les sujets sur des régulations de l'activité, envisagées ou effectuées. Des propos, comme : «Je me suis rendu compte que ... et j'ai fait.../ décidé que...», «Avant, je faisais... en pensant que..., et maintenant, je me dis que... et je fais... », témoignent de prises de conscience et de choix. Ils signifient qu'il y a eu prise de distance avec son activité et autoévaluation. Certes, l'interprétation de ces discours est délicate. Recul, distanciation, décentration ne sont que des repères pour l'observateur qui essaie de comprendre, à un moment $\mathrm{T}$, ce qui a provoqué des régulations. Les exemples, qui illustrent la description de ces processus, sont extraits de discours d'enseignants (Campanale, 1996) ou d'élèves (Campanale, 2003), qui, croisés avec d'autres données, ont montré qu'ils s'accompagnaient de régulations.

Le recul. L'autoévaluation se focalise sur des résultats (effets d'une pratique, production provisoire ou définitive) au regard des résultats envisagés, de feedback externes renvoyés par la situation, par autrui. «Qu'est-ce que j'ai fait ? Est-ce que cela marche/convient? » sont les questions que se pose plus ou moins consciemment le sujet. Ce qui est explicité, c'est la prise de conscience, éventuellement un réajustement induit par une autoévaluation, qui se limite à de l'autoconstat. Le réajustement peut ne pas se répercuter sur la conception de l'action et les moyens de réalisation, et ne pas modifier significativement la pratique ultérieure. C'est par exemple le cas de cette enseignante, qui déclare, à la fin d'une formation, avoir renoncé à punir les élèves qui ont mal réalisé le travail donné à faire à la maison, parce que cela ne servait à rien. Mais, cette modification (non négligeable pour les élèves) n'a en rien affecté sa pratique évaluative habituelle, qui continue de relever essentiellement du contrôle imposé, sans explicitation de critères d'évaluation.

La distanciation. La focalisation se déplace sur les moyens utilisés pour réaliser l'action. C'est la démarche qui est interrogée («Comment j'ai fait ?»). Le sujet se remémore la façon dont il a conduit l'activité, se la représente mentalement et l'interroge en fonction de la façon dont il voulait agir, des effets produits, de remarques/questions d'autrui, d'autres démarches possibles présentées par des pairs ou l'enseignant/le formateur. La régulation porte sur la mise en œuvre d'une ou de plusieurs procédures. Ces remaniements, plus importants que les réajustements provoqués par le simple recul vis-à-vis d'un résultat et le constat d'éléments manquants ou inefficaces, s'accompagnent d'un langage intérieur qui conduit la régulation de l'activité. C'est ce dont témoignent ces paroles d'une enseignante: «Avant, je ne voulais pas revenir sur ma pratique très spontanément ... Maintenant, je m'évalue sur le contrôle que j'ai donné. C'est à dire que ... j'ai mal posé les questions... » (Campanale, 1996, p. 309'. Elle ajoute qu'elle est davantage attentive à la compréhension des consignes par les élèves et qu'elle n'hésite plus à modifier les 
Campanale, F. (2007). Evaluation réflexive en formation professionnelle et évaluation interactive dans le8 classes. In L. Allal \& L. Mottier-Lopez (dir.), Régulation des apprentissages en situation scolaire et en formation (pp. 191-206). Bruxelles : De Boëck.

questions écrites au tableau, quand elle s'aperçoit que les élèves ne les comprennent pas.

La décentration. Le sujet sort de ses propres représentations pour saisir l'objet à travers d'autres références. Il regarde sa façon d'agir, à travers le prisme d'autrui, sous l'effet d'un autre éclairage de l'action et de la situation, de l'explicitation de conceptions qui viennent déstabiliser les siennes. Et cela l'amène à s'interroger sur la pertinence de ses propres représentations, sur ce qui l'a amené à agir ainsi. La décentration peut générer une remise en cause plus profonde de son réseau de représentations, une réorientation de ses conceptions qui refonde l'activité ultérieure. La régulation porte à la fois sur sa façon de penser l'action et sur sa façon de la conduire.

On en trouve trace dans ces propos d'une autre enseignante : «Avant, je ne pouvais pas supporter que les élèves ne travaillent pas, parce que ça me renvoyait une image négative de moi-même et je ne pouvais vraiment pas le supporter, je me mettais en colère. Maintenant, j'essaie de dire et de le ressentir et de le penser qu'il y a leur liberté ${ }^{8}$. Elle peut se révéler négativement en disant non, mais... peut être cela changera... Ça m’a changé mon regard par rapport aux élèves » (Campanale, 1996, p. 311). Ces propos manifestent une réorientation des représentations de cette enseignante, quant à son rôle et au statut de l'élève, qui se traduit par une régulation active de son attitude en classe : elle déclare ne plus les «materner », les aider à leur demande, accepter qu'ils se corrigent avant de rendre leur travail à l'aide des critères d'évaluation.

La décentration déstabilise. Les propos qui en témoignent sont un peu hachés, émaillés de redites. La régulation qui s'opère est difficile à expliciter. Aussi, on n'en trouve que rarement des traces dans les discours a posteriori des élèves sur leur apprentissage. Citons, toutefois, le cas de cette élève de 10 ans, qui ayant réorganisé sa description, déclare que, maintenant, elle pensera à organiser ce qu'elle écrit, et ajoute qu'elle se rend compte que les textes donnés comme exemples par l'enseignant sont organisés, alors qu'elle ne le voyait pas avant. Sa représentation de ce qu'est un texte a été modifiée par la régulation entreprise sur le sien, suite à une situation d'évaluation mutuelle. La régulation a contribué à un apprentissage.

La distanciation et la décentration touchent le référentiel d'action. Toutefois, plus ce qui est observé et évalué touche des représentations profondes et ancrées, plus le processus «ne peut se conduire que dans un mouvement de perpétuel aller et retour, au fil des ruses et des dérobades des sujets, et des tiroirs à double fond qui s'ouvrent lentement ou brusquement» (Ardoino \& Berger, 1986, p. 9). Aussi, l'ampleur de la prise de distance et ses effets dépendent de plusieurs facteurs :

\footnotetext{
${ }^{8}$ Il était question du désinvestissement de quelques élèves de l'étude du latin en fin d'année de $3^{\text {ème }}$ en France.
} 
Campanale, F. (2007). Evaluation réflexive en formation professionnelle et évaluation interactive dans le classes. In L. Allal \& L. Mottier-Lopez (dir.), Régulation des apprentissages en situation scolaire et en formation (pp. 191-206). Bruxelles : De Boëck.

- l'organisation de la situation d'autoévaluation, qui devrait solliciter, au-delà du recul et du constat d'écart entre un résultat et un attendu, une interrogation des représentations de l'action ;

- la motivation du sujet par rapport à son projet d'apprentissage ou de formation (Laveault, 1999);

- son sentiment d'auto-efficacité (Gombert \& Fayol, 1988; Bouffard-Bouchard, 1992) ;

- la « souplesse » cognitive du sujet.

Si l'enseignant ou le formateur peut plus ou moins jouer sur les trois derniers facteurs, par contre l'organisation des situations d'autoévaluation relève entièrement de sa responsabilité.

\section{MODALITÉS DES SITUATIONS D'AUTOÉVALUATION}

Quelles situations introduire dans la formation des enseignants pour provoquer de l'autoévaluation activant de la prise de distance ? La typologie des situations d'autoévaluation des élèves, qui est un contenu de la formation à l'évaluation, est aussi la grille de lecture à travers laquelle ils peuvent identifier celles dont ils font l'expérience en formation.

\subsection{Les modalités des situations d'autoévaluation dans les classes}

Allal (1991, 1993, 1999b) distingue :

- les situations d'autoévaluation au sens strict (l'élève tout seul évalue sa production et / ou ses procédures en fonction d'un référent externe comme une grille de critères, un questionnaire guidant la réalisation, voire des documents de référence de type dictionnaires, manuels, notes de cours...);

- les situations d'évaluation mutuelle (les élèves évaluent entre eux leurs productions et procédures en fonction d'une grille de critères) ;

- les situations de co-évaluation élève-enseignant.

Dans les pratiques de classe, le dispositif généralement adopté, dans les disciplines littéraires, organise ces situations suivant la combinaison princeps de Leselbaum (1982a \& 1982b), à savoir dans l'ordre présenté ci-dessus, avec la même grille de critères de réussite comme outil médiateur des interactions. Cette organisation et le choix de l'outil sont discutables. 
Campanale, F. (2007). Evaluation réflexive en formation professionnelle et évaluation interactive dans llo@ classes. In L. Allal \& L. Mottier-Lopez (dir.), Régulation des apprentissages en situation scolaire et en formation (pp. 191-206). Bruxelles : De Boëck.

Au niveau de l'organisation, on sait maintenant que les autoévaluations au sens strict, pratiquées en premier dans ce dispositif, produisent peu de régulations (Leselbaum, 1982b). Les élèves, centrés sur leur production, invités davantage à une autocorrection qu'à un questionnement de leur référentiel, sont mis en situation de recul, et on ne peut guère attendre plus que des corrections de surface (Genthon, 1991). Par contre, des effets positifs de l'évaluation mutuelle sur l'amélioration des productions ont été montrés (Allal \& Michel, 1993). Il conviendrait alors de privilégier les situations d'évaluation mutuelle, riches en interactions immédiates, pour des productions provisoires, qui peuvent être réorientées/réorganisées, et de réserver les situations d'autoévaluation au sens strict pour des productions terminales. On peut aussi se demander si l'investissement réclamé aux élèves par une multiplicité de situations d'évaluation ne se fait pas au détriment de l'apprentissage. Il y a un équilibre délicat à trouver.

Au niveau de l'outil, est-il pertinent d'utiliser, pour les trois situations, la même forme de grille, avec des critères de réussite à confirmer ou infirmer par un signe $(\mathrm{O} / \mathrm{N}$ ou $+/$-) ? Un tel outil est adapté à l'autocontrôle (ou contrôle mutuel) terminal. Mais, en situation d'évaluation mutuelle, il n'incite guère à l'explicitation, à la confrontation des démarches, à la distanciation, à la décentration. Des questions ouvertes relatives aux critères de réalisation essentiels ${ }^{9}$, suivies d'une rubrique «propositions d'amélioration », sollicitent davantage les interactions verbales. Elles incitent à un dialogue, au delà du constat, et peuvent alors surgir ces «mots de transition » (Jorro, 2000) entre les élèves ; chacun étant, tour à tour, «arpenteur », qui tout en questionnant l'autre se questionne, et «coryphée », qui rappelle l'exigence de la norme et suggère. C'est ce que montre l'exemple, qui suit, extrait d'un mémoire professionnel de professeurs d'école stagiaires (Vivès \& Vaufredaz, 2003). Il s'agit d'un échange entre élèves de CM1, en situation d'évaluation mutuelle d'une production écrite : la description de leur monstre.

$\begin{array}{ll}\text { Fanny : } & \text { Est-ce que tu peux imaginer mon monstre comment il est ? } \\ \text { Boris : } & \text { Moi, j'dirais pas trop. } \\ \text { Fanny : } & \text { Et qu'est-ce qui va pas ?... Et qu'est-ce qui va pas ? Moi, je pense que oui. } \\ & \text { Peut-être que j'ai pas mis la couleur mais moi, je le vois bien. } \\ \text { Boris : } & \text { Mais moi, je pense qu'il y a pas assez de choses pour dire comment il est. } \\ \text { Fanny : } & \text { Et qu'est-ce qu'il faudrait améliorer par exemple? } \\ \text { Boris : } & \text { Mettre la couleur, les petites choses, quoi. }\end{array}$

Quant aux situations de co-évaluation, quand elles ne se résument pas à un exercice formel, elles permettent aux enseignants d'engager un échange avec les élèves sur les écarts d'appréciation, qui favorise l'intercompréhension. On voit aussi émerger dans les classes des bilans de fin d'apprentissage ${ }^{10}$, conduit par l'enseignant avec un ou quelques élèves. Cet entretien enseignant-élève/s est une forme de co-

\footnotetext{
${ }^{9}$ Par exemple, plutôt : «Comment le portrait est-il organisé ? que « Le portrait est organisé : Oui ? Non? ».

${ }^{10}$ Voir la pratique fondatrice des portfolios (Allal, Wegmuller, Bonaiti-Dugerdil \& CochetKaeser, 1998).
} 
Campanale, F. (2007). Evaluation réflexive en formation professionnelle et évaluation interactive dans lek classes. In L. Allal \& L. Mottier-Lopez (dir.), Régulation des apprentissages en situation scolaire et en formation (pp. 191-206). Bruxelles : De Boëck.

évaluation, qui peut porter autant sur les processus que sur les acquisitions dont témoignent les productions. Il comporte une dimension proactive, pour des apprentissages ultérieurs.

\subsection{Les modalités des situations d'autoévaluation dans les formations d'enseignants}

En formation continue (ou développement professionnel) d'enseignants, quelles situations organiser pour provoquer autoévaluation et régulation des pratiques évaluatives en classe ? Ces situations peuvent-elles être similaires à celles que le formateur propose de mettre en ouvre avec les élèves ? Contextes et enjeux de la formation diffèrent de ceux de l'apprentissage des élèves. Les relations entre les acteurs ne sont pas empreintes de la même dissymétrie. Les formés sont des adultes, des enseignants confirmés, qui se sont engagés volontairement dans la formation, qui ont plus ou moins choisi leur formateur, ont négocié avec lui les objectifs et modalités. Des situations trop similaires à celles préconisées pour les élèves risqueraient de susciter une impression d'infantilisation. Aussi, si pratiques évaluatives en formation et pratiques évaluatives en classe devraient relever du même modèle, c'est plus des relations d'analogie que d'homothétie qui peuvent les caractériser.

Les situations, qui figurent ci-après, sont reprises de l'étude ${ }^{11}$ déjà citée et ont été réutilisées pour des actions de formation ultérieures. Elles privilégient les interactions sociales entre formés et entre formés et formateur, à propos d'objets qui sont des traces de pratiques évaluatives ou de conceptions de l'évaluation scolaire.

\subsubsection{Les évaluations interactives}

Elles sont organisées en alternance avec des apports du formateur sur les résultats des recherches en docimologie, sur ce qu'est évaluer, sur les différentes formes d'évaluation et leur place dans la séquence d'enseignement-apprentissage. L'objet inter évalué est une production témoignant d'une pratique : copies d'élèves, descriptions de séquences.

Des copies d'élèves, non encore évaluées, sont souvent le premier objet que les enseignants acceptent de soumettre au regard des autres (à défaut, le formateur peut en fournir). Il leur est demandé de préciser, en petits groupes, la ou les compétences visées par la tâche prescrite et les critères d'évaluation, de noter, de formuler les appréciations qui figureront en tête de la copie. Les résultats du travail sont ensuite confrontés collectivement, en grand groupe et un référent commun est

\footnotetext{
${ }^{11}$ Cette étude (Campanale, 1996) a montré qu'une action de formation à l'évaluation, distribuée sur trois ans pour une équipe d'enseignants de français de collège, et intégrant des évaluations de pratiques interactives, produisait davantage de changements que des actions de formation centrées sur l'appropriation d'un savoir théorique et des prescription de modalités concernant l'évaluation formative.
} 
Campanale, F. (2007). Evaluation réflexive en formation professionnelle et évaluation interactive dans le\& classes. In L. Allal \& L. Mottier-Lopez (dir.), Régulation des apprentissages en situation scolaire et en formation (pp. 191-206). Bruxelles : De Boëck.

établi, qui est à la fois interne et externe pour chacun. Les interactions verbales, qui se produisent au cours de cette élaboration, les diverses interprétations des copies des élèves font prendre conscience de la subjectivité inhérente à toute évaluation scolaire. Cela met en évidence l'intérêt de l'explicitation des critères pour évaluer rigoureusement. «Je ne savais pas comment les noter, je pataugeais, alors que maintenant je sais mieux où je vais devant un paquet de rédactions, car on a défini des critères. Donc je sais que je tiens compte essentiellement de ça, de ça, de ça et pas de tout », dit une enseignante à l'issue d'une formation (Campanale, 1996, p. 307). Mais, ce n'est pas suffisant pour que les enseignants se mettent à « voir les erreurs de leurs élèves comme des signes à déchiffrer plutôt que comme des fautes à sanctionner $\gg($ Hadji, 1992, p. 24).

La description de la séquence mise en œuvre $^{12}$, dans laquelle s'inscrit l'évaluation précédente, peut ensuite être analysée. La séquence est régulée collectivement, du point de vue de sa cohérence pédagogique, du point de vue didactique en fonction de références théoriques fournies/rappelées par le formateur et/ou les pairs. La régulation prend en compte aussi les contraintes du contexte de la classe et de l'établissement. Ce travail provoque des interactions verbales très nourries, vives parfois, au cours desquelles se confrontent les représentations des enseignants sur les savoirs à faire acquérir et sur les activités d'appropriation de ces savoirs.

$\mathrm{Au}$ formateur, il revient de questionner, de suggérer des aménagements, d'inciter à se référer à la didactique de la discipline pour anticiper sur des difficultés prévisibles, pour analyser des erreurs constatées. Il peut faire identifier les temps de la séquence où une évaluation formative non instrumentée a été mise en œuvre intuitivement, et en proposer une instrumentalisation. Il peut aussi faire repérer, à partir de l'analyse de la compétence complexe visée par la séquence, décomposée en composantes élémentaires ou «règles de production »(Grégoire, 1999), quelles modalités d'évaluation formative pourraient être introduites. Cette nouvelle version de la séquence sera alors testée avec des élèves par d'autres enseignants que les auteurs de la première version. Ils en rendront compte lors de la séance de formation suivante, pour valider la nouvelle version, proposer des modifications, des variantes et rapporter des effets perçus sur les apprentissages. Plus que les résultats des recherches, ce sont les tentatives d'évaluation mutuelle entre élèves, essayées et jugées positives par leurs collègues qui convainquent ceux qui hésitent.

Le formateur intervient directement au niveau des éclairages théoriques et indirectement par les formalisations a posteriori des séquences élaborées en cours de formation. En effet, une grande part lui revient dans ces formalisations, que les enseignants n'ont ni assez de temps, ni toujours les mots pour les faire. C'est dans ces va-et-vient entre les formalisations analysées et révisées au cours des séances de formation, révisées dans les intervalles par les formés puis le formateur, que se

\footnotetext{
${ }^{12}$ Crahay (1989), dans une note de synthèse sur la possibilité de changer sa façon d'enseigner, soulignait déjà l'importance de l'analyse a priori et a posteriori de la préparation ou planification.
} 
Campanale, F. (2007). Evaluation réflexive en formation professionnelle et évaluation interactive dans le 3 classes. In L. Allal \& L. Mottier-Lopez (dir.), Régulation des apprentissages en situation scolaire et en formation (pp. 191-206). Bruxelles : De Boëck.

précisent les notions, que surgissent les interrogations.

Les enseignants renâclent parfois à une planification rigoureuse des séquences. Ils ont, d'abord, des difficultés à distinguer compétences, tâches et critères. De plus, construire de nouvelles séquences leur demande un travail important de recherche d'informations sur le contenu et de transposition didactique. L'énergie consacrée à ce travail s'accompagne souvent d'une exigence moindre dans la rigueur de la programmation. C'est plutôt en deuxième année de formation, que les séquences sont davantage formalisées, surtout si elles deviennent objets d'évaluation entre pairs. Au fil de la formation, se constitue ainsi un réservoir commun de séquences formalisées, comprenant un cadrage général et des variantes, des supports pour l'apprentissage et des outils pour l'évaluation formative et sommative. Ce réservoir permet alors aux enseignants d'alterner des séquences très formalisées et des séquences plus informelles, qui laissent place à de l'improvisation en fonction de l'actualité de la classe ou de l'actualité sociale. La motivation des enseignants a besoin d'un espace d'invention pour tirer parti d'un événement, de phases de découverte d'un contenu nouveau qui renforce leur culture disciplinaire.

\subsubsection{Les bilans de formation accompagnés par le formateur}

Ce sont des autobilans écrits de formation, rétroactifs et proactifs, qui ponctuent les séances. Ils s'écrivent en réponse à des questions du type : Qu'est-ce que j'ai appris, compris ? Qu'est-ce que je veux approfondir? Quelles modalités d'évaluation formative ai-je essayées ? Quels en ont été les effets? Qu'est-ce que je reprendrai/ne reprendrai pas et pourquoi ? Ces bilans individuels donnent lieu à une synthèse fournie en retour par le formateur lors de la séance de formation suivante. Ils ont comme fonction de permettre une prise de conscience des évolutions par les acteurs (formés et formateur).

En fait, les formés répondent au questionnaire d'auto-bilan plus pour faire plaisir au formateur que pour s'autoévaluer, car leur projet dans ces formations, au départ, est plus d'acquérir des techniques d'évaluation qui aident les élèves à apprendre que de se changer eux-mêmes. Aussi, les auto-bilans sont davantage source d'informations pour le formateur que pour eux. Ils permettent d'identifier les difficultés, les résistances, ce sur quoi les formés sont prêts à avancer, et ce n'est pas forcément dans la direction souhaitée par le formateur. Toutefois, la synthèse des auto-bilans nourrit un échange entre formés et formateur sur le sens que prend la formation collective et individuelle. C'est dans l'échange collectif, encore en situation d'interactions verbales, que chacun s'exprime davantage sur ses évolutions, sa nouvelle façon de concevoir sa pratique. Cela renforce les prises de conscience, interroge «la volonté d'aider qui en définitive installe l'activité évaluative dans un registre formatif » (Hadji, 1997, p. 19). C'est à cette occasion surtout que se manifestent des discours autoévaluatifs sur les pratiques, du type: «Avant je faisais..., et maintenant...», «J'ai pris conscience que...», «Je me suis rendu compte que ...», «Maintenant, je me dis que...». Ces paroles sur ses pratiques relèvent autant d'un discours pour autrui que d'un «dialogue de soi à soi » suivant 
Campanale, F. (2007). Evaluation réflexive en formation professionnelle et évaluation interactive dans les classes. In L. Allal \& L. Mottier-Lopez (dir.), Régulation des apprentissages en situation scolaire et en formation (pp. 191-206). Bruxelles : De Boëck.

l'expression de Nunziati (1990). Certes, «le Dit construit a posteriori n'équivaut pas au Fait qu'il prétend reprendre ; il exerce cependant une influence réelle et formatrice sur des décisions à venir, en ce qu'il est un facteur de mise en cohérence » (Weisser, 1998, p.101).

Dans l'organisation des situations d'autoévaluation, par ses consignes, le choix des documents fournis comme références, des outils pour évaluer (critères de réalisation, questionnaires interactifs...), le formateur favorise plus ou moins telle ou telle prise de distance. Néanmoins, la façon dont chaque enseignant en formation utilise la situation, l'ampleur de sa prise de distance et des régulations qu'il effectue, dépendent du sujet, de sa personnalité, de son histoire et du contexte de travail.

\section{PROBLÈMES LIÉS AUX CONTEXTES DE TRAVAIL}

Des formations qui rassemblent les caractéristiques que nous avons recensées comme facteurs de changements de pratiques, provoquent des essais modestes mais effectifs d'évaluation mutuelle, alors même que les représentations des enseignants restent encore confuses, au niveau des notions. Les suggestions des formateurs sont prises en compte, plus sous l'effet d'une dynamique collective que sur la foi des résultats des recherches. Peut-on alors penser que les modalités de formation sont plus puissantes que les contenus eux-mêmes? On sait que la relation établie dans la communication englobe le contenu, qui est perçu à travers le cadre qu'elle installe. Faire vivre des situations d'évaluation interactives de leurs pratiques à des enseignants en formation crée une culture commune, qui est un levier puissant pour innover.

Toutefois, dans les classes, la généralisation de démarches d'évaluation formative pour réguler les apprentissages se heurte à des difficultés récurrentes. On sait que les contextes de travail influent sur la transformation des acquis de la formation en compétences nouvelles (Meignant, 1997). En quoi les tutelles nationales $^{13}$ et les établissements favorisent-ils ou entravent-ils les pratiques d'évaluation formative, les modalités de régulations d'apprentissage ?

Certes, d'une façon générale, les institutions de tutelle incitent les enseignants à se former à la pratique de l'évaluation formative, par des déclarations liminaires aux programmes d'études, en finançant des formations continues. Mais, l'évaluation formative qu'elles promeuvent est le plus souvent du type de celle proposée par Bloom (1988) dans la pédagogie de la maîtrise, où l'enseignant prend des informations sur les difficultés et réussites des élèves pour renvoyer des feedback pertinents et mettre en place des exercices de remédiation. La régulation des démarches d'apprentissage, à travers des évaluations mutuelles entre élèves, autre

\footnotetext{
${ }^{13}$ L'expression « tutelles nationales » renvoie aux collectifs ou individus chargés dans les systèmes éducatifs de rédiger les textes de cadrage pour orienter les pratiques pédagogiques dans les classes et/ou chargés de les évaluer.
} 
Campanale, F. (2007). Evaluation réflexive en formation professionnelle et évaluation interactive dans les classes. In L. Allal \& L. Mottier-Lopez (dir.), Régulation des apprentissages en situation scolaire et en formation (pp. 191-206). Bruxelles : De Boëck.

versant de l'évaluation formative, est occultée dans les discours institutionnels. Aussi, ce qui prévaut encore, dans les préoccupations des enseignants, c'est le contrôle des performances et de leur progression, plus que la prise en charge par les élèves de la régulation de leurs apprentissages.

D’une façon générale, l'évaluation-contrôle reste présente en arrière-fond dans la société scolaire. Cela est accentué par la permanence de la note chiffrée comme moyen privilégié de communication des résultats de l'évaluation. Etablissements, voire parents, réclament aux enseignants l'attribution de notes fréquentes, à dates fixes, alors même que des apprentissages ne sont pas forcément terminés. En outre, la notation intervient comme moyen de pression dans la négociation du contrat didactique (Chevallard, 1986). Et, dans les classes difficiles, avec des élèves « rétifs », indulgence ou sévérité dans l'évaluation reste un moyen tacite de «police scolaire » (Merle, 1998), auquel les enseignants continuent de recourir, même sans illusion sur son efficacité, même si l'institution affirme qu'il ne faut pas confondre évaluation du travail personnel des élèves et punitions relatives à leur comportement scolaire (Bulletin officiel de l'éducation nationale française, 2000).

Toutefois, en France, la mise en place récente des projets à réaliser par un groupe d'élèves accompagnés par une équipe pluridisciplinaire d'enseignants (travaux personnels encadrés/itinéraires de découverte/projet personnel à caractère professionnel) est une mesure prometteuse, si elle s'installe dans la durée. En effet, le carnet de bord, qui accompagne la conception et la réalisation du projet, objet d'évaluation formative, est un moyen de communication entre élèves et enseignant susceptible de provoquer de l'autoévaluation, de la métacognition et de la régulation proactive et rétroactive des apprentissages. Les enseignants, encore peu outillés pour ces démarches, réclament des formations. Dans la même perspective, émergent, en Suisse, les pratiques d'évaluation de projets de productions réalisées en groupe, à l'incitation de chercheurs (Bain, 2000). Mais, subsiste le problème de la façon dont les directions des établissements facilitent et valorisent le travail des enseignants qui s'engagent dans ces projets de travail d'équipe.

Une autre voie prometteuse est celle ouverte par Allal (2002) qui, en se référant à l'apprentissage situé, propose «d'aménager» le contrôle écrit en y introduisant des phases où l'élève peut en interaction avec d'autres réguler son travail, en somme où, au cours du contrôle, il continue à apprendre au lieu de faire seulement la preuve, en solo, de ses acquisitions. Quelle sera la position des institutions face à une telle proposition qui remet en cause l'épreuve traditionnelle du contrôle des connaissances, qui juge plus des acquisitions que des compétences ?

L'appropriation par les enseignants en exercice de nouvelles perspectives, ouvertes par les chercheurs sur les démarches de régulations interactives des apprentissages, nécessite une acculturation à ces démarches. Cela peut se faire par la réflexion sur l'évaluation et l'expérience de la régulation de ses pratiques au cours d'actions de formation. Mais la prise en compte de l'influence des contextes de 
Campanale, F. (2007). Evaluation réflexive en formation professionnelle et évaluation interactive dans llo6 classes. In L. Allal \& L. Mottier-Lopez (dir.), Régulation des apprentissages en situation scolaire et en formation (pp. 191-206). Bruxelles : De Boëck.

travail sur la mise en oeuvre de ces démarches dans les classes semble encore insuffisante. Ne conviendrait-il pas de développer des recherches pédagogiques visant à résoudre des problèmes pratiques dans les contextes où ils se posent (Van der Maren, 1999), associant chercheurs, formateurs et praticiens ? 
Campanale, F. (2007). Evaluation réflexive en formation professionnelle et évaluation interactive dans lę classes. In L. Allal \& L. Mottier-Lopez (dir.), Régulation des apprentissages en situation scolaire et en formation (pp. 191-206). Bruxelles : De Boëck.

\section{RÉFÉRENCES}

Allal, L. (1985). Stratégies d'évaluation formative : conceptions psychopédagogiques et modalités d'application. In L. Allal, J. Cardinet \& P. Perrenoud (Ed.), L'évaluation formative dans un enseignement différencié (pp. 130-156). Berne : Peter Lang.

Allal, L. (1991). Vers une pratique de l'évaluation formative : matériel de formation continue des enseignants. Bruxelles : De Boeck.

Allal, L., Bain, D. \& Perrenoud, P. (Ed.). (1993). Didactique du français et évaluation formative. Neuchâtel : Delachaux et Niestlé.

Allal, L. (1993). Régulations cognitives : quelle place pour l'élève dans l'évaluation formative ? In L. Allal, D. Bain \& P. Perrenoud (Ed.), Evaluation formative et didactique du français (pp. 81-98). Neuchâtel : Delachaux et Niestlé.

Allal, L. \& Michel, Y. (1993). Autoévaluation et évaluation mutuelle en situation de production écrite. In L. Allal, D. Bain \& P. Perrenoud (Ed.), Evaluation formative et didactique du français (pp. 239-264). Neuchâtel : Delachaux et Niestlé.

Allal, L. (1999). Impliquer l'apprenant dans le processus d'évaluation: promesses et pièges de l'autoévaluation. In C. Depover \& B. Noël (Ed.), L'évaluation des compétences et des processus cognitifs (pp. 35-56). Bruxelles : De Boeck.

Allal, L. (2001). Situated cognition and learning : From conceptual frameworks to classroom investigations. Revue suisse des sciences de l'éducation, 23(3), 407-421.

Allal, L., Wegmuller, E., Bonaiti-Dugerdil, S. \& Cochet-Kaeser, F. (1998). Le portfolio dans la dynamique de l'entretien tripartite. Mesure et évaluation en éducation, 20 (3), 5-31.

Altet, M. (2000). L'analyse de pratiques : une démarche de formation professionnalisante ? Recherche et formation, 30, 25-41.

Ardoino, J. (1993). L'approche multiréférentielle (plurielle) des situations éducatives et formatives. Pratiques de formation, 25-26, 15-34.

Ardoino, J. \& Berger, G. (1986). L'évaluation comme interprétation. Journal de la formation continue \& de l'EAO, 208, 7-10.

Bain, D. (2000). De l'évaluation aux compétences : mise en perspectives de pratiques émergentes. In J. Dolz \& E. Ollagnier (Ed.), L'énigme de la compétence en éducation (pp. 129-145). Bruxelles : De Boeck.

Bloom, B. S. (1988). Le problème des deux sigmas : la recherche de méthodes d'enseignement en groupe aussi efficaces que le préceptorat. In M. Huberman (Ed.), Assurer la réussite des apprentissages scolaires? Les propositions de la pédagogie de la maîtrise (pp. 44-83). Neuchâtel : Delachaux et Niestlé.

Bonniol, J. J. \& Vial, M. (1997). Les modèles de l'évaluation :textes fondateurs avec commentaires. Bruxelles : De Boeck.

Bouffard-Bouchard, T. (1992). Relation entre le savoir stratégique, l'évaluation de soi et le sentiment d'autoefficacité, et leur influence dans une tâche de lecture. Enfance, 46 (1-2), 63-78.

Bulletin officiel de l'éducation nationale, $\mathrm{n}^{\circ} 8$ du 13 juillet 2000 : http://www.education.gouv.fr/bo/2000/special8/proced.htm

Butera, F., Gardair, E., Maggi, J. \& Mugny, G. (1998). Les paradoxes de l'expertise : influence sociale et (in)compétence de soi et d'autrui. In J. Baillé, J. Py \& A. Somat (Ed.), Psychologie sociale et formation professionnelle : propositions et regards cliniques (pp. 111-123). Rennes : Presses Universitaires de Rennes.

Campanale, F. (1996). L'autoévaluation, facteur de transformations des conceptions et des pratiques. Etudes d'actions de formation continue des enseignants dans le domaine de l'évaluation. Thèse de doctorat en Sciences de l'éducation. Université Pierre-Mendès-France (Grenoble II).

Campanale, F. (1997). Autoévaluation et transformation de pratiques pédagogiques. Mesure et évaluation en éducation, 20 (1), 1-24.

Campanale, F. (1998). Modélisation des processus d'autoévaluation. Pédagogies, revue du département des sciences de l'éducation de l'université de Louvain, 12, 13-19. 
Campanale, F. (2007). Evaluation réflexive en formation professionnelle et évaluation interactive dans le8 classes. In L. Allal \& L. Mottier-Lopez (dir.), Régulation des apprentissages en situation scolaire et en formation (pp. 191-206). Bruxelles : De Boëck.

Campanale, F. (1999). D’un modèle de l'autoévaluation aux situations d'évaluation partagée dans la formation initiale des enseignants. In C. Fabre-Cols \& E. Triquet (Ed.) Actes du 2ème colloque international recherche(s) et formation des enseignants. IUFM de Grenoble (CD-Rom).

Campanale, F. (2003, septembre). L'évaluation mutuelle interrogée par les principes d'efficacité et d'équité. Papier présenté au $16^{\circ}$ colloque international de l'Adméé-Europe «Evaluation entre efficacité et équité », Université de Liège.

Cardinet, J. (1986). Evaluation scolaire et pratique. Bruxelles : De Boeck.

Chevallard, Y. (1986). Vers une analyse didactique des faits d'évaluation. In J.-M. De Ketele (Ed.), L'Evaluation : approche descriptive ou prescriptive? (pp. 31-59). Bruxelles : De Boeck.

Chevallard, Y. (1991). La transposition didactique. Grenoble : La pensée sauvage.

Crahay, M. (1989). Contraintes de situation et interactions maître-élève : changer sa façon d'enseigner, est-ce possible ? Revue française de pédagogie, 88, 67-94.

De Montmollin, M. (1996). Savoir travailler : le point de vue de l'ergonome. In J.-M. Barbier (Ed.), Savoirs théoriques et savoirs d'action (pp. 189-199). Paris : Presses Universitaires de France.

Fayol, M. (1998). Apprendre : éléments pour une approche cognitive. Recherche et formation, 27, 4150.

Genthon, M. (1991). Communiquer : quoi, pour quoi faire et comment, dans un processus d'évaluation? In J. Weiss (Ed.), L'évaluation: problème de communication (pp. 175-188). Cousset (Fribourg): DelVal.

Gombert, J. E. \& Fayol, M. (1988). Auto-contrôle par l'enfant de ses réalisations dans des tâches cognitives. Revue française de pédagogie, 82, 47-59.

Grégoire, J. (1999). Que peut apporter la psychologie cognitive à l'évaluation formative et à l'évaluation diagnostique ? In C. Depover \& B. Noël (Ed.), L'évaluation des compétences et des processus cognitifs (pp. 17-56). Bruxelles : De Boeck.

Hadji, C. (1992). L'évaluation des actions éducatives. Paris : Presses Universitaires de France.

Hadji, C. (1997). L'évaluation démystifiée. Paris : ESF.

Huberman, M. (1986). Un nouveau modèle pour le développement professionnel des enseignants. Revue française de pédagogie, 75, 5-15.

Jorro, A. (2000). L'enseignant et l'évaluation: des gestes évaluatifs en question. Bruxelles : De Boeck.

Laveault, D. (1999). Autoévaluation et régulation des apprentissages. In C. Depover \& B. Noël (Ed.), L'évaluation des compétences et des processus cognitifs (pp. 57-79). Bruxelles : De Boeck.

Leselbaum, N. (1982a). Autonomie et auto-évaluation. Paris: Economica.

Leselbaum, N. (1982b). Le travail autonome: premier essai d'évaluation d'une innovation pédagogique. Revue française de pédagogie, 59, 9-23.

Malglaive, G. (1994). Les rapports entre savoir et pratique dans le développement des capacités d'apprentissage chez les adultes. Education permanente, 119, 125-133.

Meignant, A. (1997). Manager la formation (4º́d. actualisée et enrichie). Rueil-Malmaison : Editions Liaisons.

Merle, P. (1998). Sociologie de l'évaluation scolaire. Paris : Presses Universitaires de France

Mugny, G., Quiamzade, A., Pigière, D., Dragulescu, A. \& Buchs, C. (2002). Self-competence, interaction style and expert social influence : Toward a correspondence hypothesis. Swiss Journal of Psychology, 61, 153-166.

Noël, B., Romainville, M. \& Wolfs, J. L. (1995). La métacognition : facettes et pertinence du concept en éducation, Revue française de pédagogie, 112 (3), 47-56.

Nunziati, G., (1990). Pour construire un dispositif d'évaluation formatrice. Cahiers pédagogiques : apprendre, 280, 48-64.

Perrenoud, P. (1993). Touche pas à mon évaluation! Pour une approche systémique du changement. Mesure et évaluation en éducation, 16 (1-2), 107-132.

Perrenoud, P. (1996). Le travail sur l'habitus dans la formation des enseignants : analyse des pratiques et prise de conscience. In L. Paquay, M. Altet, E. Charlier \& P. Perrenoud (Ed.), Former des enseignants professionnels (pp. 181-207). Bruxelles : De Boeck .

Perrenoud, P. (1999). De quelques compétences du formateur-expert. http://www.unige.ch/fapse/SSE/teachers/perrenoud/php_main/php_1999/1999_15.html

Piaget, J. (1974a). La prise de conscience. Paris : Presses Universitaires de France. 
Campanale, F. (2007). Evaluation réflexive en formation professionnelle et évaluation interactive dans lleg classes. In L. Allal \& L. Mottier-Lopez (dir.), Régulation des apprentissages en situation scolaire et en formation (pp. 191-206). Bruxelles : De Boëck.

Piaget, J. (1974b). Réussir et comprendre. Paris : Presses Universitaires de France.

Prost, A. (1985). Eloge des pédagogues. Paris : Seuil.

Romainville, M. (1993). Savoir parler de ses méthodes. Bruxelles : De Boeck.

Talyzina, N. F. (Ed.) (1980). De l'enseignement programmé à la programmation de la connaissance. Presses Universitaires de Lille.

Vandenberghe, R. (1986). Le rôle de l'enseignant dans l'innovation en éducation. Revue française de pédagogie, 75, 17-26.

Van der Maren, J.M. (1999). La recherche appliquée en pédagogie. Bruxelles : De Boeck.

Weissier, M. (1998). L'existence précède l'essence. Recherche et formation, 27, 93-102.

Vivès, M. \& Vaufredaz, A.S. (2003). Les effets des situations d'autoévaluation sur la prise de conscience de l'apprentissage chez l'élève. Mémoire professionnel PE2, IUFM de Grenoble.

Vial, M. (1995). Nature et fonction de l'autoévaluation dans le dispositif de formation. Revue française de pédagogie, 112, 69-76.

Vygotsky, L.S. (1985). Pensée et Langage. Paris : Editions sociales. 